\title{
IceCube searches for neutrino emission from galactic and extragalactic sources
}

\author{
Tomasz Jan Palczewski for the IceCube Collaboration ${ }^{1,2, a}$ \\ ${ }^{1}$ Department of Physics and Astronomy, The University of Alabama, Tuscaloosa, AL, 35487 \\ ${ }^{2}$ https://icecube.wisc.edu/collaboration/authors
}

\begin{abstract}
The IceCube Neutrino Observatory, located near the geographic South Pole, is currently the world's largest neutrino telescope. IceCube is principally designed to detect high-energy neutrinos from galactic and extragalactic sources. The detector comprises more than a cubic-kilometer of glacial ice instrumented with 86 vertical strings, each with 60 optical sensors, and a square-kilometer array at the surface. IceCube sensors detect Cherenkov radiation from charged particles produced in all neutrino flavor interactions in the ice. We discuss the recent results from searches for high-energy neutrinos, including the first detection of a diffuse flux of extraterrestrial origin with energies between about $30 \mathrm{TeV}$ and $2 \mathrm{PeV}$. The events with energies above $1 \mathrm{PeV}$ are the highest energy neutrinos ever observed.
\end{abstract}

\section{Introduction}

High-energy neutrinos are expected to be produced in astrophysical objects by the decays of charged hadrons, mainly pions, produced in accelerated particle interactions with nearby radiation or gas [1-3]. Neutrinos, being mostly massless and weakly interacting particles, can propagate virtually unimpeded through matter. Neutrinos are also not deflected in galactic or extra-galactic magnetic fields and therefore point back to the site of production, tentatively permitting the study of the dynamics and interiors of source high red-shift objects like gamma-ray bursts and active galactic nuclei. Neutrinos with energies larger than several $100 \mathrm{TeV}$ are especially of interest because in this energy region the atmospheric background falls rapidly. The first indications of astrophysical neutrinos were found by the IceCube Collaboration while the IceCube detector was still under construction [4-6]. In this contribution we present a search for high-energy neutrinos interacting with the IceCube detector in the data-taking period between 2010 and 2013 [6-9].

\section{IceCube Detector}

The IceCube detector, located near the geographic South Pole, is currently the world's largest neutrino telescope (see Fig. 1). The detector consists of 86 cables ("strings"), each instrumented with 60 Digital Optical Modules (DOMs). The DOM consists of a 10-inch photomultiplier tube (PMT), calibration light sources, and digitizing electronics [10]. DOMs are deployed at depths between $1450 \mathrm{~m}$ and $2450 \mathrm{~m}$ below the surface. The hor- izontal distance between most IceCube string is on average $125 \mathrm{~m}$ with the DOMs vertically spaced on a string at $17 \mathrm{~m}$. Eight strings near the center are more densely spaced with $42 \mathrm{~m}-72 \mathrm{~m}$ horizontal spacing and $7 \mathrm{~m}$ vertical spacing. Most DOMs on these eight strings contain PMTs with $35 \%$ higher quantum efficiency than standard IceCube DOMs. These eight strings, combined with the twelve nearest IceCube strings, make up DeepCore sub detector array. IceCube also includes 81 surface stations, called IceTop.

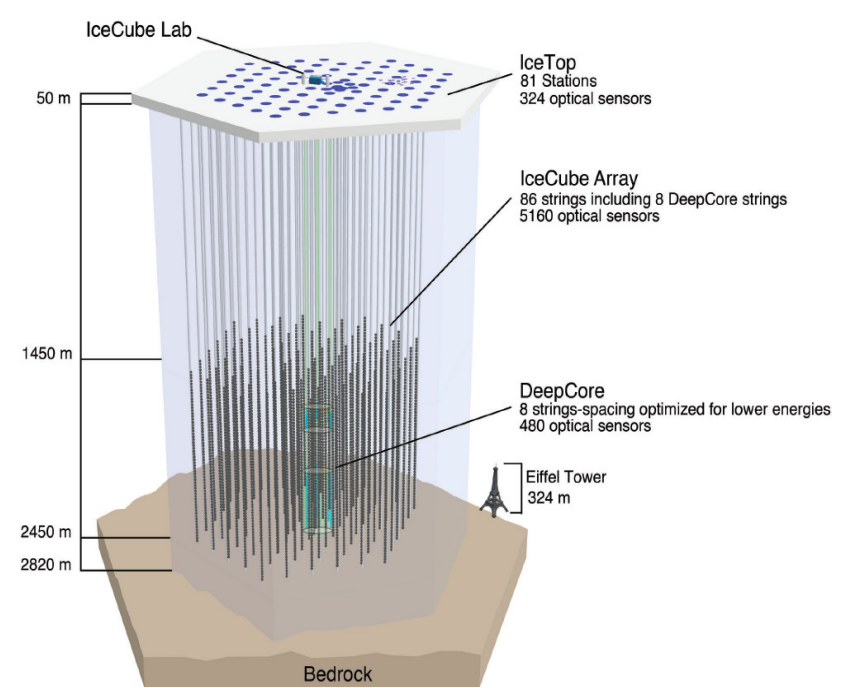

Figure 1. A sketch of the the IceCube detector showing the inice array and locations for the DeepCore subarray and IceTop stations.

\footnotetext{
a e-mail: tomas.j.palczewski@ua.edu
} 


\section{Detection Principle}

When neutrinos interact in ice via charged-current (CC) or neutral-current (NC) processes, they produce charged particles that may emit Cherenkov radiation which can be detected by the DOMs. The characteristic light pattern (topology) of the Cherenkov light provides information about the energy, direction and flavor of the parent neutrino. In IceCube one can distinguish between the three different types of light pattern topologies from neutrino interactions, classified as:

- Track-like events (see Fig. 2), which have a good angular resolution $\left(<1^{\circ}, 50 \% C L\right)$ but limited energy resolution. These events are produced mainly from the $v_{\mu}$ $\mathrm{CC}$ interactions.

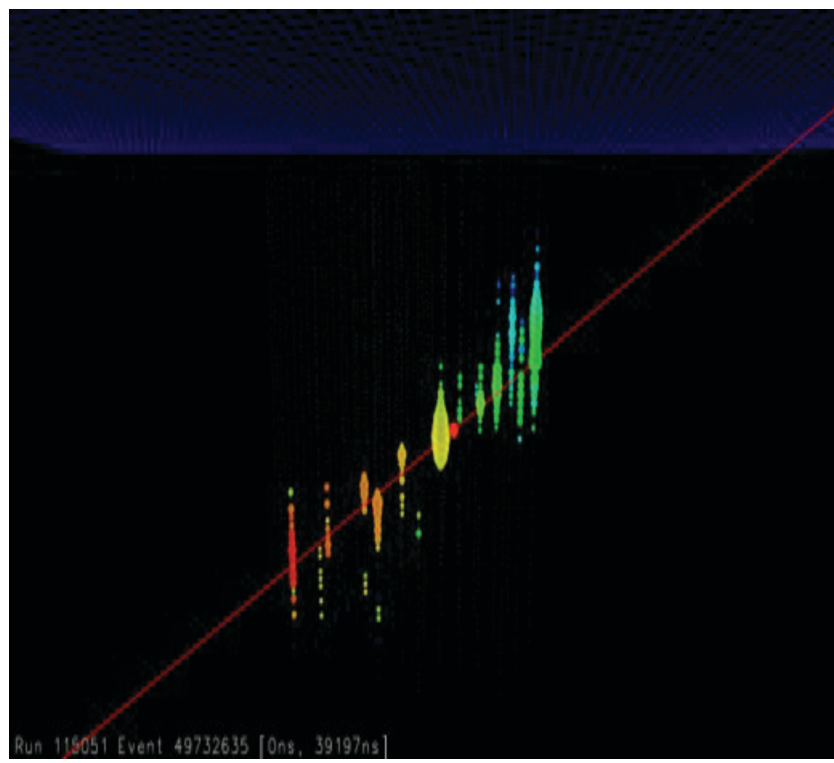

Figure 2. Track-like event topology.

- Cascade-like events (see Fig. 3), which have a good energy resolution ( $\sim 10 \%$ at high energies) but limited angular resolution $\left(>10^{\circ}, 50 \% C L\right)$. They are described by a distinct point-like spherical signature in the detector volume. This pattern can be seen as a result of $v_{e}, v_{\mu}$, $v_{\tau} \mathrm{NC}$ and $v_{e} \mathrm{CC}$ interactions.

- Composite events (see Fig. 4), which are mixture of track-like and cascade-like events. This pattern has been produced by $v_{\mu} \mathrm{CC}$ interactions and is expected for $v_{\tau}$ $\mathrm{CC}$ interactions (not yet observed).

Note that in Fig. 2, 3 and 4 the size of the individual DOMs displays the relative measured charge while the color represents the photon arrival times with red being early and blue late in time. The timing information and charge deposition permit reconstruction of event direction and deposited energy. The uncertainties connected with the modeling of natural glacial ice and with the determination of the absolute energy scale are the largest contributors to the systematic errors of the energy and angular reconstruction [11].

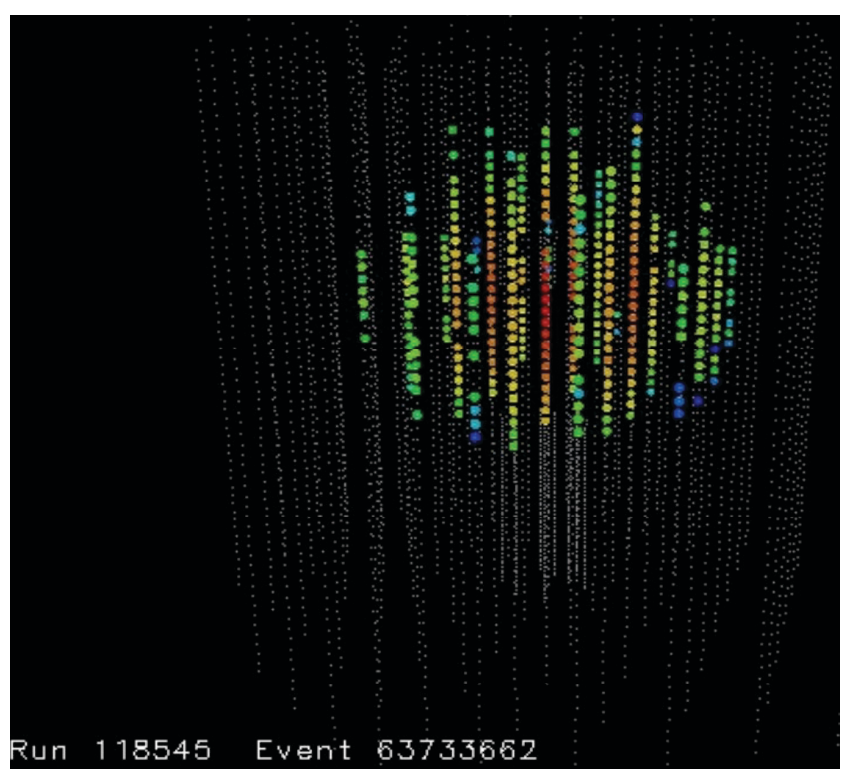

Figure 3. Cascade-like event topology.

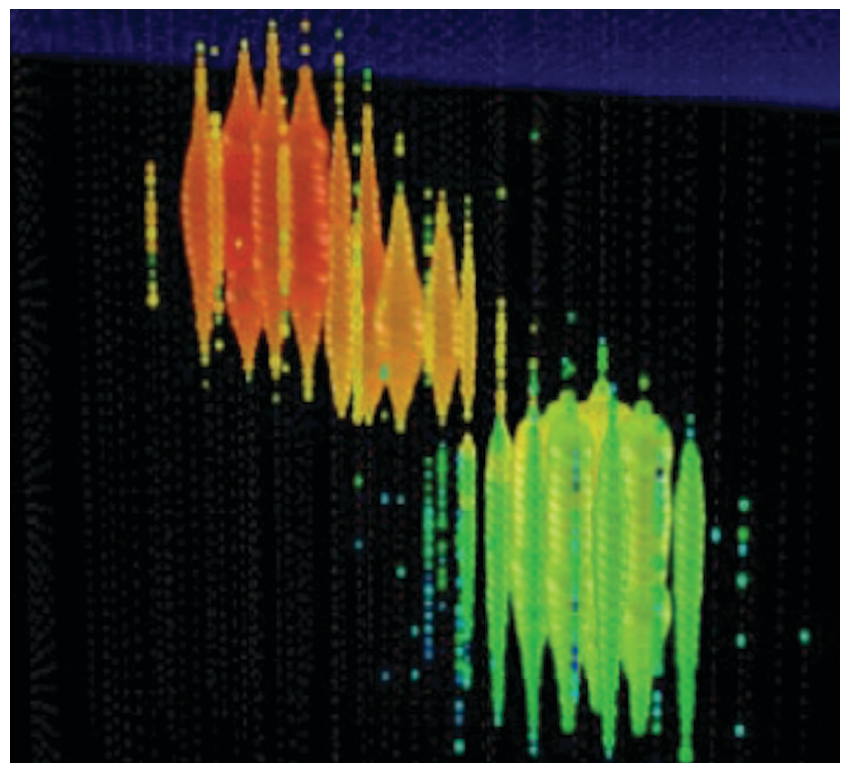

Figure 4. Composite event topology.

\section{IceCube Detector Performance}

Evaluating the long-term performance of the IceCube array is a crucial element of the data analysis. To facilitate this, each DOM includes 12 LEDs flashers that are used as calibration sources [12]. Additionally, one string also holds a nitrogen laser with absolute calibration that serves as a standard candle. Together, these calibration devices are used to perform timing, charge amplitude, and geometry calibrations for the compete array, and also make it possible to extract the optical properties of the ice. From this monitoring, the detector has shown excellent stability with over $98 \%$ of the DOMs in continuous operation.

It is also possible to characterize the angular resolution and absolute pointing of the detector. As an example, cosmic rays incident on the Moon will be absorbed creating a deficit, or shadow, for energetic muons in the Moon's 
direction. Using the IceCube 40- and 59-string configurations (IC40 and IC59, respectively), this Moon shadow analysis was performed for $\mathrm{TeV}$ energy events, and has been observed to high significance $(>6 \sigma)$ [13]. In Fig. 5, the Moon shadow in the centre region of the plot for the IC59 has an observed location within 0.2 degrees of the expected position. Fig. 6 shows the shadow position to-

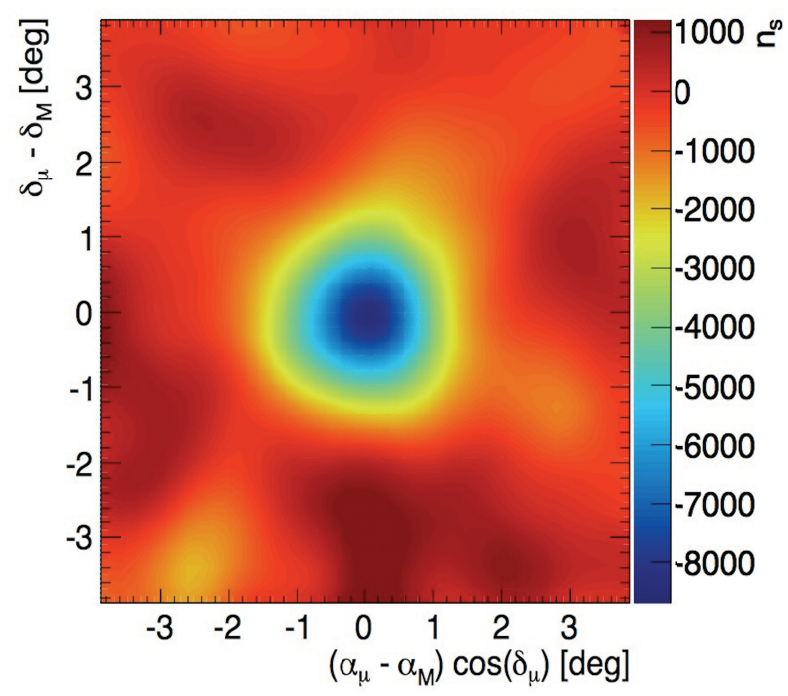

Figure 5. The contour maps of the number of signal events in the data $\left(n_{s}\right)$ in the $(\Delta \alpha, \Delta \delta)$ coordinate system for on-source regions of the IC59 dataset [13]. The $\Delta \alpha$ is a right ascension difference and $\Delta \delta$ is a declination difference with respect to the nominal Moon position.

gether with $1 \sigma, 2 \sigma$, and $3 \sigma$ contours.

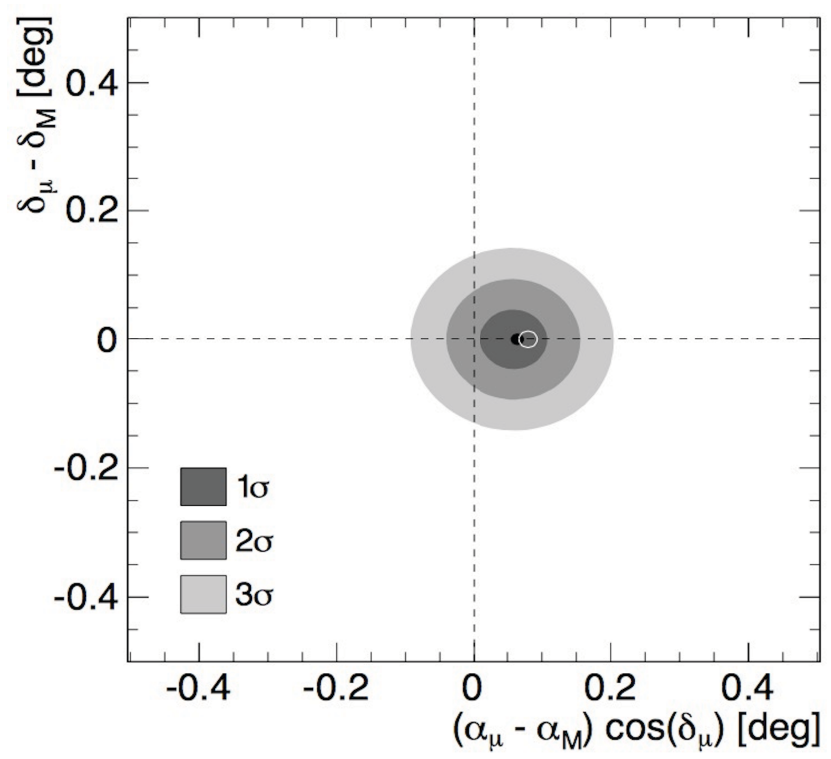

Figure 6. The shadow position together with $1 \sigma, 2 \sigma$, and $3 \sigma$ contours for IC59 data [13]. The reconstructed position for the Moon shadow from the maximum likelihood analysis (black point) and expected position of the Moon shadow after accounting for magnetic deflection (white circle) are superimposed.
In addition, the average angular resolution may be estimated by applying a Gaussian function to the shadow profile. The $1 \sigma$ width of the Moon shadow was found to be $0.7^{\circ}$, which is in good agreement with the estimated angular resolution from the simulation studies of down-going muons.

\section{First Observation of PeV-energy Neutrinos}

IceCube detected two cascade-like events with an estimated deposited energies of about $1 \mathrm{PeV}$ (the highest energy neutrinos observed at the time) in a search for ultrahigh energy (UHE) neutrinos using data corresponding to 615.9 days of effective live time [6]. These two events, representing an excess of $2.8 \sigma$ above expected backgrounds (see Fig. 7), were the first indication of a highenergy astrophysical neutrino flux.

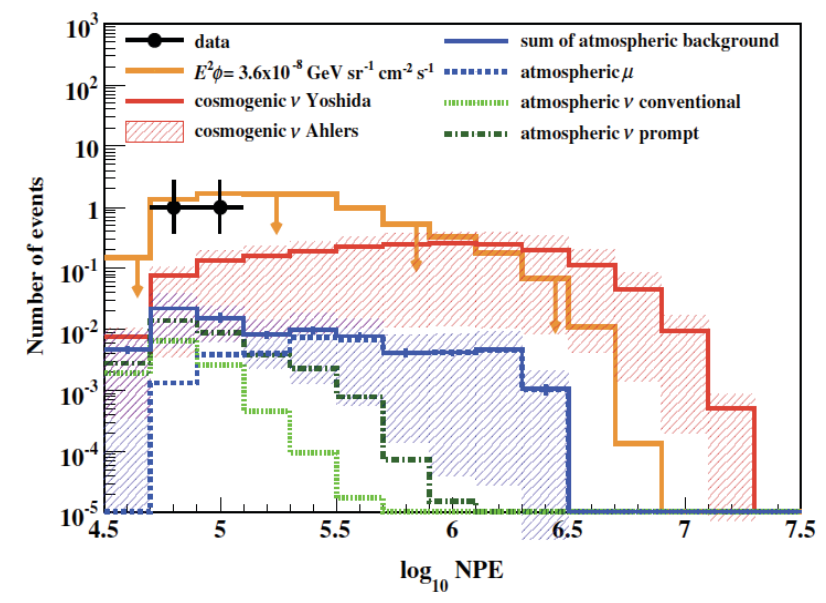

Figure 7. The number of observed photoelectrons for 615.9 days of live time after final selection cuts [6]. Shown is the experimental data (black points) with the simulated backgrounds, including atmospheric muons (dashed blue); conventional atmospheric neutrinos (dotted light green); prompt atmospheric neutrinos (dotted-dashed green). The red line represents the cosmogenic neutrino model [14]. The error bars on the line and the shaded regions are the statistical and systematic uncertainties, respectively.

\section{High-Energy Astrophysical Neutrinos in Three Years of IceCube Data}

A follow-up analysis to the ultra-high energy search described above was performed for high-energy neutrino events interacting in the detector fiducial volume in 988 days of effective detector live time [8]. Starting events in the detector were selected in order to suppress the background from down-going atmospheric muons. This selection was realized by applying the anti-coincidence muon veto in the outer layers of the detector, requiring that fewer than 3 of the first 250 detected photoelectrons (PE) occur in the detector boundary. The energy threshold was 
lowered (sensitivity down to energies near $30 \mathrm{TeV}$ ) in order to characterize the flux that leads to the observed PeV events from the UHE search. A minimum of 6000 detected $\mathrm{PE}$ was required to ensure sufficient statistics were available for an efficient veto. The atmospheric neutrino background rates were estimated using measurements of the northern-hemisphere $v_{\mu}$ spectrum [5]. In the full 988 day sample, 37 events were detected with the dominant component being cascade-like events from the southern sky (down-going), see Fig. 8. An additional analysis was

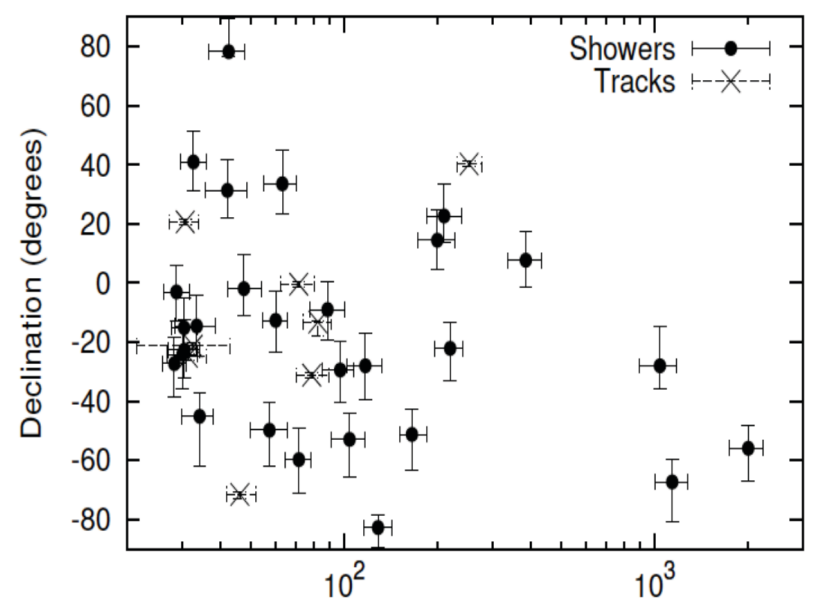

Deposited EM-Equivalent Energy in Detector (TeV)

Figure 8. Arrival angles and deposited energies of 37 events detected in the full 988-day sample [8].

developed to study both the northern sky and track-like events in the sample (see Sect. 7).

The number of expected atmospheric neutrino background events from model assumptions and cosmic ray muon events are $6.6_{-1.6}^{+5.9}$ and $8.4 \pm 4.2$, respectively. Applying the energy and angular distributions of the events, see Fig. 9, an atmospheric-only explanation for the flux is disfavored by $5.7 \sigma$. The expected suppression in the northern hemisphere from Earth absorption of the highest energy neutrinos is also observed. The data are described well by the expected backgrounds and a hard astrophysical isotropic neutrino flux. For a benchmark $\mathrm{E}^{-2}$ spectrum, the best-fit per-flavor fux normalization under $1: 1: 1$ flavor ratio assumption is $\mathrm{E}^{2} \Phi_{\nu}(\mathrm{E})=0.95 \pm 0.3 * 10^{-8} \mathrm{GeV} \mathrm{cm}^{-2} \mathrm{~s}^{-1} \mathrm{sr}^{-1}$.

Fig. 10 shows the event directions in Galactic coordinates. No evidence of neutrino emission from point-like or extended sources was found. The most significant cluster is that close to the Galactic Centre with a p-value of $7 \%$.

\section{Diffuse Astrophysical Muon Neutrinos with Two Years of IceCube Data}

A very important confirmation of the previous result has now been detected in a diffuse $v_{\mu}$ analysis. The up-going muon track diffuse search on two years of data (May 2010 to May 2012) has shown an excess of events above background expectations at energies above approximately
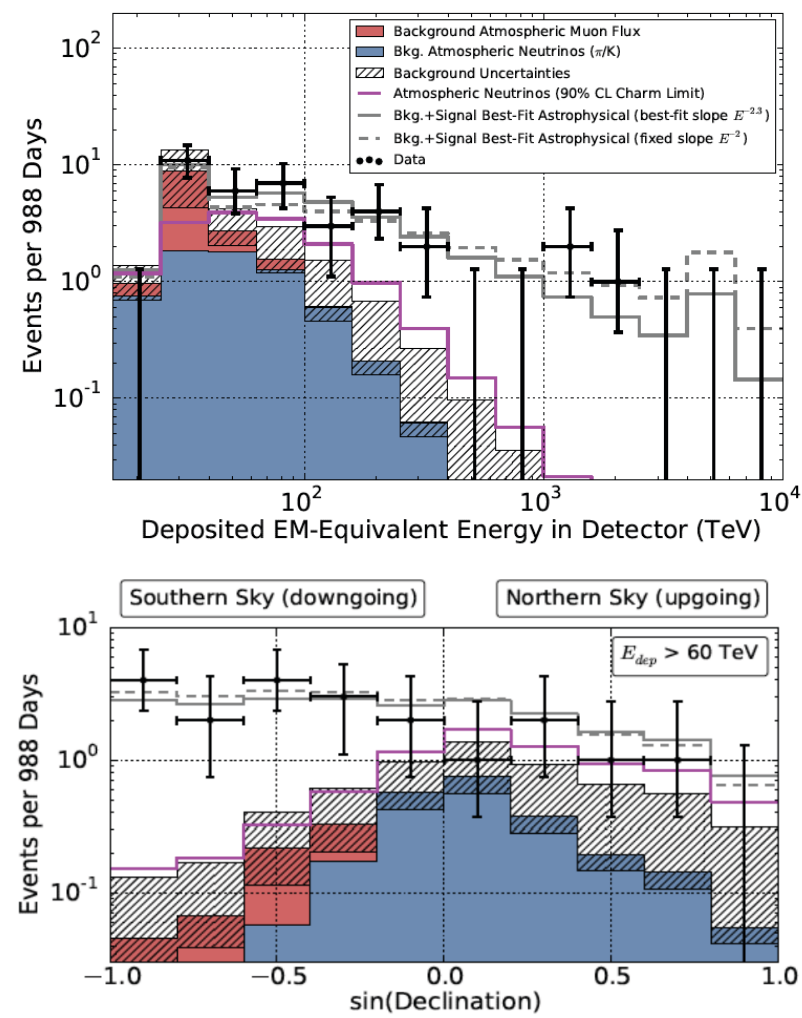

Figure 9. The energy (top) and declination (bottom) distributions of 37 observed events in the full 988-day sample compared to background expectations [8].

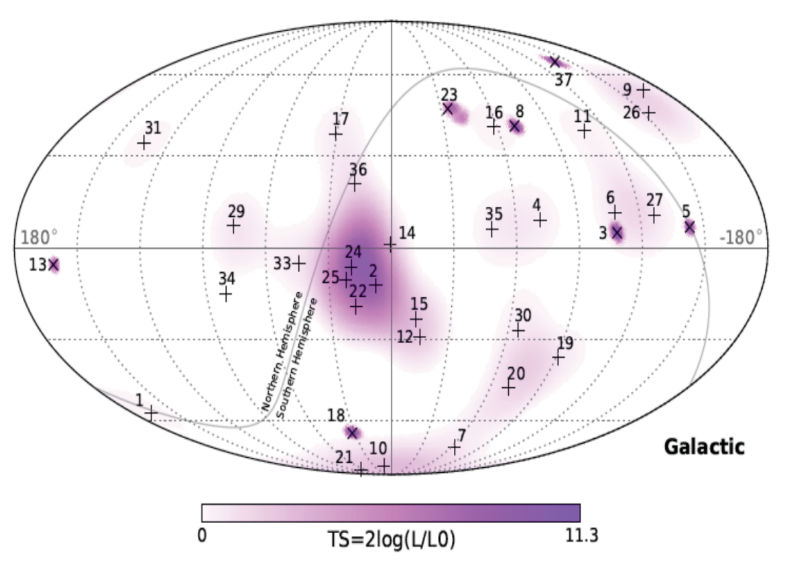

Figure 10. Arrival directions of the events in Galactic coordinates [8]. Shower-like events are marked with + and track-like events with $\mathrm{x}$. Event IDs match those in the supplement material of [8] and are time ordered. The grey line denotes the equatorial plane. Colors show the test statistic (TS) for the point source clustering test at each location.

$100 \mathrm{TeV}$ as shown in Fig. 11 for the energy-loss distribution which is correlated with the initial neutrino energy. 


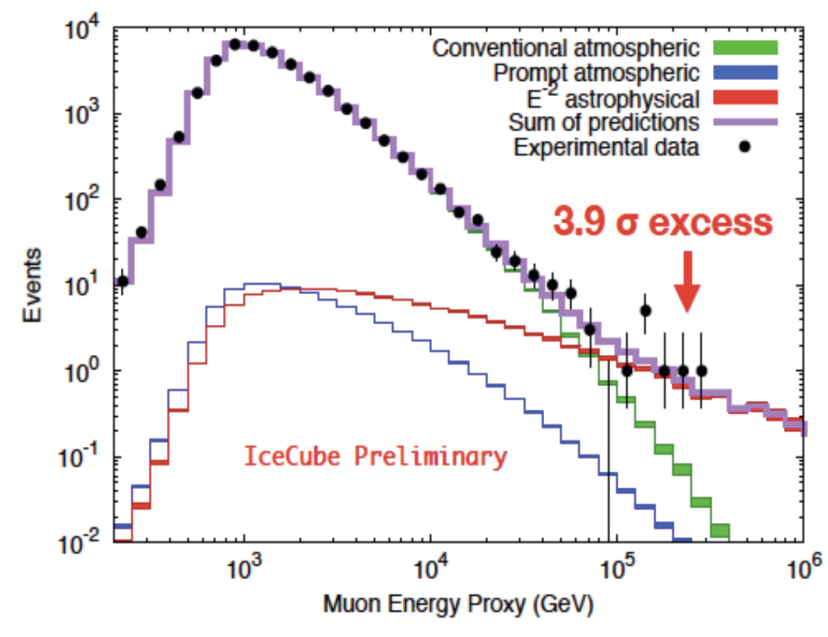

Figure 11. The preliminary reconstructed muon energy loss distribution.

Since this final event sample has minimal overlap with the sample discussed in Sect. 6, this measured excess may be treated as an independent confirmation of a high-energy astrophysical neutrino flux $3.9 \sigma$ above the atmospheric-only explanation. For a benchmark $\mathrm{E}^{-2}$ spectrum, the best-fit per-flavor fux normalization under $1: 1: 1$ flavor ratio assumption is $\mathrm{E}^{2} \Phi_{v}(\mathrm{E})=1.01 \pm 0.35^{*} 10^{-8} \mathrm{GeV} \mathrm{cm}^{-2} \mathrm{~s}^{-1} \mathrm{sr}^{-1}$.

\section{Conclusions}

IceCube has reported the first significant detection of highenergy astrophysical neutrinos and an independent confirmation analysis. The reconstructed spectrum is compatible with a power law. The source of this flux has not yet been observed, but further observations with data from the current detector is now under analysis. In addition, a planned future detector array is now being evaluated that would significantly increase the sensitivity for this measurement.

\section{References}

[1] S. H. Margolis, D. N. Schramm, R. Silberberg, Astrophys. J. 221, 990 (1978).

[2] F. W. Stecker, Astrophys. J. 228, 919 (1979).

[3] W. Michalak, W. J. Wdowczyk, A. W. Wolfendale, J. Phys. G 16, 1917 (1990).

[4] M. G. Aartsen et al. (IceCube Collaboration), Phys. Rev. D 89, 102001 (2014).

[5] M. G. Aartsen et al. (IceCube Collaboration), Phys. Rev. D 89, 062007 (2014).

[6] M. G. Aartsen et al. (IceCube Collaboration), Phys. Rev. Lett. 111, 021103 (2013).

[7] M. G. Aartsen et al. (IceCube Collaboration), Science 342, 1242856 (2013).

[8] M. G. Aartsen et al. (IceCube Collaboration), Phys. Rev. Lett. 113, 101101 (2014).

[9] C. Weaver (for the IceCube Collaboration), APS April Meeting Vol. 59, R8.00001, (2014).

[10] M. G. Aartsen et al. (IceCube Collaboration), JINST 9, P03009 (2014).

[11] R. Abbasi et al. (IceCube Collaboration), Nucl. Instrum. Methods A 601, 294 (2009).

[12] J. Kiryluk, M. V. D’Agnostino, S. R. Klein, C. Song, D. R. Williams (for the IceCube Collaboration), arXiv 0711.0353 [astro-ph], 95 (2007).

[13] M. G. Aartsen et al. (IceCube Collaboration), Phys. Rev. D 89, 102004 (2014).

[14] S. Yoshida, M. Teshima, Prog. Theor. Phys. 89, 833 (1993). 